\title{
UNCERTAINTY ANALYSIS OF DECOMPOSING POLYURETHANE FoAM"
}

\author{
Michael L. Hobbs and Vicente J. Romero \\ Sandia National Laboratories, Albuquerque, New Mexico $87185^{\dagger}$
}

\begin{abstract}
Sensitivity/uncertainty analyses are necessary to determine where to allocate resources for improved predictions in support of our nation's nuclear safety mission. Yet, sensitivity/uncertainty analyses are not commonly performed on complex combustion models because the calculations are time consuming, CPU intensive, nontrivial exercises that can lead to deceptive results. To illustrate these ideas, a variety of sensitivity/uncertainty analyses were used to determine the uncertainty associated with thermal decomposition of polyurethane foam exposed to high radiative flux boundary conditions. The polyurethane used in this study is a rigid closed-cell foam used as an encapsulant. Related polyurethane binders such as Estane are used in many energetic materials of interest to the JANNAF community.
\end{abstract}

The complex, finite element foam decomposition model used in this study has 25 input parameters that include chemistry, polymer structure, and thermophysical properties. The response variable was selected as the steady-state decomposition front velocity calculated as the derivative of the decomposition front location versus time. An analytical mean value sensitivity/uncertainty (MV) analysis was used to determine the standard deviation by taking numerical derivatives of the response variable with respect to each of the 25 input parameters. Since the response variable is also a derivative, the standard deviation was essentially determined from a second derivative that was extremely sensitive to numerical noise. To minimize the numerical noise, $50-\mu \mathrm{m}$ element dimensions and approximately $1-\mathrm{msec}$ time steps were required to obtain stable uncertainty results.

As an alternative method to determine the uncertainty and sensitivity in the decomposition front velocity, surrogate response surfaces were generated for use with a constrained Latin Hypercube Sampling (LHS) technique. Two surrogate response surfaces were investigated: 1) a linear surrogate response surface (LIN) and 2) a quadratic response surface (QUAD). The LHS techniques do not require derivatives of the response variable and are subsequently relatively insensitive to numerical noise. To compare the LIN and QUAD methods to the MV method, a direct LHS analysis (DLHS) was performed using the full grid and timestep resolved finite element model. The surrogate response models (LIN and QUAD) are shown to give acceptable values of the mean and standard deviation when compared to the fully converged DLHS model.

\section{INTRODUCTION}

Figure 1.A shows a comparison between an X-ray and a 2-D finite element simulation of an $8.8-\mathrm{cm}$ diameter right circular cylinder of polyurethane foam encapsulating a solid $3.8-\mathrm{cm}$ diameter by $6.4-\mathrm{cm}$ long right circular cylinder of 304 stainless steel. The top of the foam has been exposed to a radiation heat source. The $X$-ray and model show exceptional agreement when comparing the shape of the decomposition front after a 10-minute exposure to an incident flux of $25-W / \mathrm{cm}^{2}$. The 2-D serial simulation required 11 days and 16 hours of CPU time on a 400-Mhz SUN-Ultra-2 using 11,209 elements. Even with massively parallel computers, an uncertainty analysis for this CPU-intensive model is difficult. A simple representation of the complex model is needed for efficient sensitivity analysis. Figure 1.B shows a single row of ele-

A) Decomposition front shape

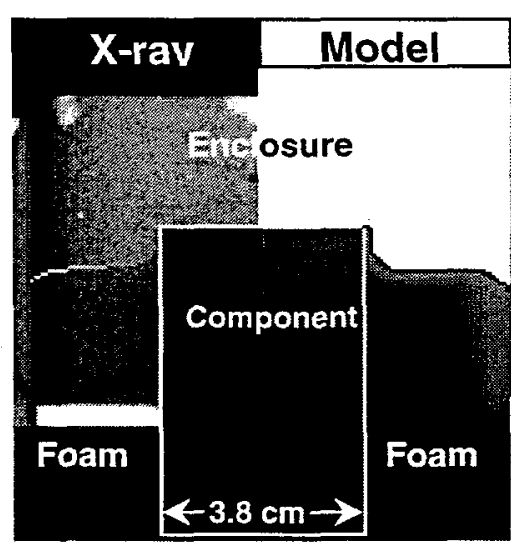

Fig. 1. A) Original and B) simplified model ments with a radiation boundary condition. This simple 1-D model will be used in lieu of the complex 2-D model to do sensitivity/uncertainty analysis. Gartling et al., ${ }^{1}$ Chu et al., ${ }^{2}$ and Hobbs et al. ${ }^{3}$ give details about the finite element model, decomposition experiments, and decomposition model, respectively.

Approved for public release; distribution is unlimited.

t This work performed at Sandia National Laboratories. Sandia is a multiprogram laboratory operated by Sandia Corporation, a Lockheed Martin Company, for the U.S. Department of Energy under contract DE-ACO4-94AL85000. 


\section{DISCLAIMER}

This report was prepared as an account of work sponsored by an agency of the United States Government. Neither the United States Government nor any agency thereof, nor any of their employees, make any warranty, express or implied, or assumes any legal liability or responsibility for the accuracy, completeness, or usefulness of any information, apparatus, product, or process disclosed, or represents that its use would not infringe privately owned rights. Reference herein to any specific commercial product, process, or service by trade name, trademark, manufacturer, or otherwise does not necessarily constitute or imply its endorsement, recommendation, or favoring by the United States Government or any agency thereof. The views and opinions of authors expressed herein do not necessarily state or reflect those of the United States Government or any agency thereof. 


\section{DISCLAIMER}

Portions of this document may be illegible in electronic image products. Images are produced from the best available original document. 


\section{NUMERICAL ISSUES}

The response variable for the 1-D analysis was chosen as the steady-state decomposition front velocity, calculated as the derivative of the decomposition front location versus the elapsed time for element death. Figure 2.A shows the front location calculated using $25-\mu \mathrm{m}$ elements exposed to a $1,000^{\circ} \mathrm{C}$ radiative temperature. The decomposition front is located at the centroid of the element that is exposed to the radiation boundary. Elements are removed from the computational domain when the condensed mass fraction drops below 1 percent.

Figure 2.B shows decomposition front velocities calculated using various element sizes. The average time steps, decomposition front velocities, and CPU times for the solutions shown in Fig. 2 are given in Table 1. The larger time steps (large $\Delta t_{\text {ave }}$ ) are based on the Gartling et al. auto time stepping scheme with an integration convergence tolerance of $10^{-4}$. The solutions obtained with smaller time steps (small $\Delta t_{\text {ave }}$ ) are based on the same convergence tolerance but are constrained to satisfy the following derivative smoothness criterion:

$$
\Delta t \leq 0.003 \Delta x / V
$$

where $\Delta t, \Delta x$, and $V$ represent the time step, element size, and the decomposition front velocity, respectively. In Fig. 2.B, the small time step solutions are smooth (solid lines, $0.5-\mathrm{mm}$ and $1-\mathrm{mm}$ ) compared to the noisy solutions obtained with larger time steps. The small time step curves for 25- and 50- $\mu \mathrm{m}$ element solutions overlie each other, so the less costly $50-\mu \mathrm{m}$ element using fixed time step results are effectively converged. The noisy velocities in Fig. 2.B resulted from taking excessively large time steps for the instantaneous derivative calculation. Rather than imposing the time step constraint in Eq. (1), the integration convergence tolerance could have been decreased with the same expense of increased CPU cost.

The numerical noise is related to the discrete removal of elements from the computational domain. Figure 3 shows the temperature gradient in element No. 20 for a $650^{\circ} \mathrm{C}$ radiation temperature using $0.6-\mathrm{mm}$ elements. The first 20 elements are also shown in Fig. 3 centered about the time of "element death." The noise is associated with the death of neighboring elements and is likely the source of the sensitivity to the computational time step.

The remainder of this paper will focus on determination of the standard deviation of the decomposition front velocity and ranking the importance of the 25 model input parameters using the MV, LIN, QUAD, and DLHS models. Fully grid and time-step converged solutions using 50- $\mu \mathrm{m}$ elements and $\sim 1$-msec time steps were used in the MV and DLHS analyses. Larger time steps were used in the LIN and QUAD methods. All methods give similar results since the response function is nearly linear, however, the LIN method was computationally less expensive.

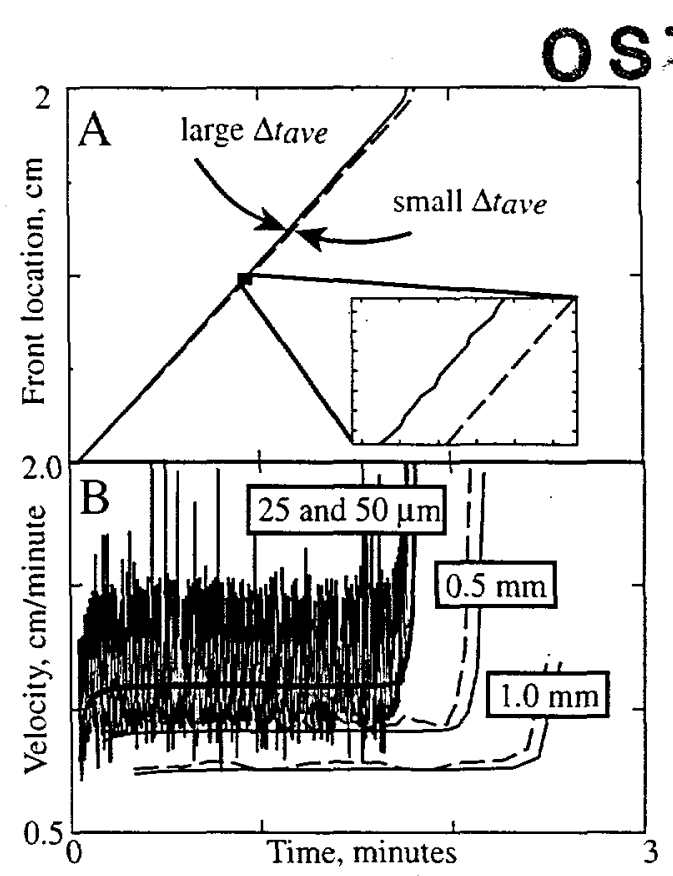

Fig. 2. A) Location $B$ ) velocities

Table 1. Time step and CPU time

\begin{tabular}{|c|l|l|}
\hline Grid & Large $\Delta t_{\text {ave }}$ (auto) & Small $\Delta t_{\text {ave }}$ (Eq. 1) \\
\hline \hline 25 & $\Delta t_{\text {ave }}=0.066 \mathrm{sec}$ & $\Delta t_{a v e}=0.00041 \mathrm{sec}$ \\
$\mu \mathrm{m}$ & $V_{\text {ave }}=1.12 \mathrm{~cm} / \mathrm{min}$ & $V_{\text {ave }}=1.10 \mathrm{~cm} / \mathrm{min}$ \\
& $\mathrm{CPU}=13 \mathrm{~min}$ & $\mathrm{CPU}=5 \mathrm{hrs}$ \\
\hline 50 & $\Delta t_{\text {ave }}=0.076 \mathrm{sec}$ & $\Delta t_{\text {ave }}=0.00082 \mathrm{sec}$ \\
$\mu \mathrm{m}$ & $V_{\text {ave }}=1.11 \mathrm{~cm} / \mathrm{min}$ & $V_{\text {ave }}=1.09 \mathrm{~cm} / \mathrm{min}$ \\
& $\mathrm{CPU}=6 \mathrm{~min}$ & $\mathrm{CPU}=1 \mathrm{hr}$ \\
\hline 0.5 & $\Delta t_{\text {ave }}=0.14 \mathrm{sec}$ & $\Delta t_{\text {ave }}=0.0095 \mathrm{sec}$ \\
$\mathrm{mm}$ & $V_{\text {ave }}=0.94 \mathrm{~cm} / \mathrm{min}$ & $V_{\text {ave }}=0.91 \mathrm{~cm} / \mathrm{min}$ \\
& $\mathrm{CPU}=70 \mathrm{sec}$ & $\mathrm{CPU}=110 \mathrm{sec}$ \\
\hline 1.0 & $\Delta t_{\text {ave }}=0.202 \mathrm{sec}$ & $\Delta t_{\text {ave }}=0.023 \mathrm{sec}$ \\
$\mathrm{mm}$ & $V_{\text {ave }}=0.77 \mathrm{~cm} / \mathrm{min}$ & $V_{\text {ave }}=0.76 \mathrm{~cm} / \mathrm{min}$ \\
& $\mathrm{CPU}=120 \mathrm{sec}$ & $\mathrm{CPU}=130 \mathrm{sec}$ \\
\hline
\end{tabular}

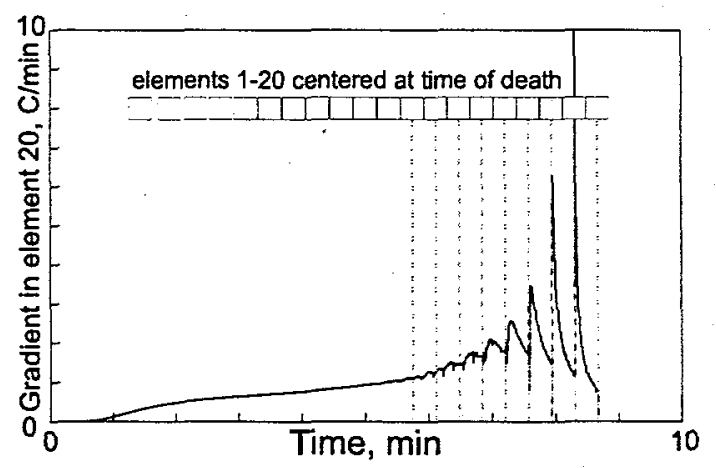

Fig. 3. Temperature gradient 


\section{MOdel INPUt PARAMETERS}

The means, $\bar{m}_{i}$ and standard deviations, $\sigma_{i}$, of the foam decomposition model's 25 input parameters are given in Table 2. The input parameters include: the initial density and temperature of the foam $\left(\rho_{0}\right.$ and $T_{0}$ ), the temperature-dependent thermal conductivity and specific heat of the foam ( $k$ and $C_{p}$ ), the coordination number of the polymer lattice $(s+1)$, the initial lattice bridge population $\left(L_{0}\right)$, the reaction enthalpy of the foam $\left(h_{r}\right)$, the emissivity of the foam $(\varepsilon), 16$ activation energies $\left(E_{i}\right)$, and the average standard deviation of all the activation energies $\left(E_{\sigma}\right)$. The input parameters are assumed to be independent random variables that are normally distributed. Some of the mean input parameters - e.g. activation energies - were determined by optimizing predicted mass loss to experimental Thermal Gravimetric Analysis (TGA) data. Other mean input parameters, such as thermal conductivity and specific heat, were measured at Purdue's Thermophysical Properties Research Laboratory. The standard deviations for the input parameters were taken to be approximately 10 percent of the mean value for all parameters except for the initial temperature and the activation energies, which are assumed to be within $3^{\circ} \mathrm{C}$ and $300 \mathrm{Kcal} / \mathrm{mol}$, respectively.

Table 2. Means and standard deviations of input parameters

\begin{tabular}{|c|c|c|c|c|c|c|c|c|c|c|c|c|c|}
\hline & $p_{0}, g / c c$ & $T_{0}$ & $\boldsymbol{K}$ & $C_{p}{ }^{n}$ & $s+1$ & $L_{0}$ & $h_{n} \mathrm{cal} \mathrm{cm}^{3}$ & $\varepsilon$ & $E_{t}^{b}$ & $E_{2}^{b}$ & $E_{3}^{b}$ & $E_{4}^{b}$ & $E_{5}^{b}$ \\
\hline $\bar{m}_{i}$ & 0.353 & 300 & 1 & 1 & 2.8 & 0.78 & 15 & 0.8 & 48.8 & 50.5 & 49.7 & 50.2 & 49.4 \\
\hline$\sigma_{i}$ & 0.0252 & 3 & 0.1 & 0.1 & 0.2 & 0.05 & 1.5 & 0.05 & 0.3 & 0.3 & 0.3 & 0.3 & 0.3 \\
\hline & $E_{6}^{b}$ & $E_{7}^{b}$ & $E_{8}^{b}$ & $E_{g}^{b}$ & $E_{10}^{b}$ & $E_{11}{ }^{\circ}$ & $E_{12}^{b}$ & $E_{13}{ }^{b}$ & $E_{14}{ }^{6}$ & $E_{15}^{b}$ & $E_{16}^{b}$ & $E_{\sigma}^{b}$ & \\
\hline $\bar{m}_{i}$ & 49.1 & 50.6 & 49.5 & 51.2 & 49.4 & 50.3 & 49.7 & 50.6 & 50.7 & 50.2 & 49.7 & 3.97 & \\
\hline$\sigma_{i}$ & 0.3 & 0.3 & 0.3 & 0.3 & 0.3 & 0.3 & 0.3 & 0.3 & 0.3 & 0.3 & 0.3 & 0.05 & \\
\hline
\end{tabular}

a The temperature-dependent thermal conductivity and heat capacity were multiplied by a dimensionless factor.

${ }^{b}$ Activation energies in $\mathrm{Kcal} / \mathrm{mol}$

\section{Mean Value Sensitivity/Uncertainty Analysis}

In the mean value (MV) method, the mean decomposition front velocity, $\mu_{\mathrm{V}}$, and the standard deviation of the decomposition front velocity, $\sigma_{V}$, can be determined from a simple Taylor series expansion of the front velocity, $V(\bar{\zeta})$, about the mean of the individual random variables or input parameters, $\zeta_{i}$ by neglecting higher order terms as follows:

$\mu_{V}=\left.V(\bar{\zeta})\right|_{\bar{\zeta}=\bar{m}}$

$\sigma_{V}^{2}=\sum_{i=1}^{n}\left[\left.\sigma_{i} \frac{\partial V(\bar{\zeta})}{\partial \zeta_{i}}\right|_{\bar{\zeta}=\bar{m}}\right]^{2}$

Equation (2) is a single-sample approximation of the mean decomposition front velocity calculated with the finite element model with all input parameters equal to the mean values, $\bar{m}$. In Eq. (3), $\sigma_{v}$ is the standard deviation of the decomposition front velocity (response variable) and $\sigma_{i}$ is the standard deviation of the $f^{\text {th }}$. input parameter (random variable). The derivatives in Eq. (3) were obtained using a central differencing technique with a finite difference step size of 0.001 times the mean input parameter. For each temperature, 51 function evaluations (two for each random variable plus one evaluation using the mean input values) were required to obtain the derivatives for the 25 random variables.

The relative importance of each input variable to the uncertainty in the decomposition front velocity can be determined from the sensitivity coefficients, $\gamma_{i}$, defined as:

$\gamma_{i}=\frac{\sigma_{i}}{\sigma_{V}} \times \frac{\partial V(\bar{\zeta})}{\partial \zeta_{i}}$,

where

$-1 \leq \gamma_{i} \leq 1$ 
and

$\sum_{i=1}^{n} \gamma_{i}^{2}=1$

The input variables that contribute the most to the uncertainty in the decomposition front velocity also have the largest absolute sensitivity values. The sign of the sensitivity coefficients indicate that an increase in the input parameter causes an increase in the response function. A negative sensitivity coefficient indicates that an increase in the input parameter causes a decrease in the response function. The square of the sensitivity coefficient multiplied by 100 gives an importance factor, $100 \times \gamma_{i}^{2}$, that can be used to easily identify which input variables are important.

Figure 4 shows the mean decomposition front velocity with a $1.96-\sigma$ band and the standard deviation of the front velocity, the importance of the most significant input parameters, and various sensitivity coefficients as a function of the radiative boundary temperature. The most important input parameters are the emissivity of the foam followed by the foam heat capacity and density. The lattice coordination number and the activation energies associated with Reactions 5,6, and 9 are also important factors in Fig. 4.B. An increase in emissivity will cause the decomposition front velocity to increase, whereas an increase in density or heat capacity will cause the decomposition front velocity to decrease.
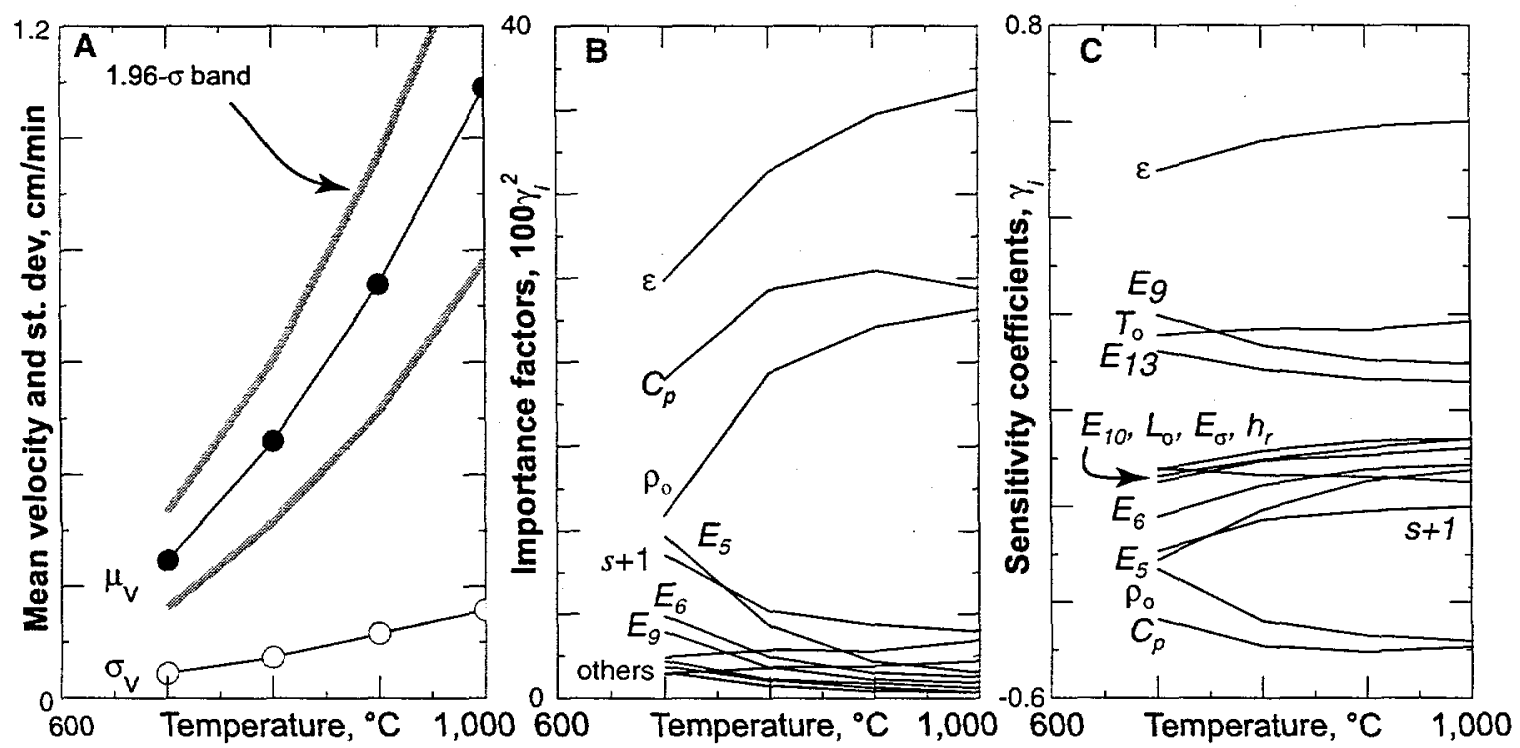

Figure 4. A) Velocity, B) importance factors, and C) sensitivity coefficients

\section{Sensitivity/Uncertainty ANalysis Using SuRrogate Response Surfaces}

Surrogate response surfaces can also be used for sensitivity/uncertainty analysis by using Latin Hypercube Sampling (LHS) techniques. Similar to the MV technique discussed in Section 2, surrogate response surfaces should only be used when the response is nearly linear. Highly nonlinear response functions require advanced techniques as discussed by Robinson. ${ }^{4}$ One advantage of using surrogate response surfaces is that the derivative of the response variable is not needed for the sensitivity/uncertainty analysis. Another important feature of the surrogate-LHS technique is that the method typically requires fewer functional evaluations using the finite element model than the traditional MV methods and gives similar accuracy.

The objective of the surrogate LHS uncertainty analysis, as discussed by Romero and Bankston, ${ }^{5,6}$ is to adequately represent the system response over the relevant parameter space with a simple analytical function that can be used efficiently in an LHS simulation. For example, the simplest representation of the decomposition front velocity as a function of the 25 input parameters is the following linear response function: 
$V_{L I N}=a_{0}+\sum_{j=1}^{25} a_{i}\left(\zeta_{i}-\bar{m}_{j}\right)$

where $V_{L I N}$ is the linear approximation of the decomposition front velocity fit to results from the 1-D finite element model. This "level 1" response surface can be used to evaluate the derivative in Eq. (3) to give

$$
\sigma_{V, L I N}^{2}=\sum_{i=1}^{25}\left(\sigma_{i} a_{i}\right)^{2}
$$

The $a$ coefficients can easily be determined with 26 functional evaluations using the full finite element model. One velocity evaluation is determined using the mean of all the input parameters. The remaining 25 velocity evaluations are determined using the mean of all the input parameters except for the $f^{t h}$ parameter. The $f^{t h}$ parameter is taken at the mean value plus some fraction of the standard deviation of the input parameter, $\tau \sigma_{i}$. Unfortunately, an established technique for selecting $\tau$ does not exist. In this work, a $\tau$ value of 2 was used as recommended by Romero. ${ }^{7}$ Guan and Melcher ${ }^{8}$ recently showed that different values of $\tau$ for nonlinear response surfaces could give significantly different sensitivity results. However, since the response surface in the current paper is sufficiently linear, a value of 2 is adequate.

The a coefficients can be determined as follows:

$a_{0}=V_{0}=\left.V(\bar{\zeta})\right|_{\bar{\zeta}=\overline{\bar{m}}}$

and

$a_{i}=\left(V_{i}^{+\tau \sigma}-V_{o}\right) /\left(\tau \sigma_{i}\right)$ for $i=1,25$,

where $V_{0}$ is the decomposition front velocity calculated with the 1-D finite element model using the mean value of each input parameter; $V_{i}^{+\tau \sigma}$ is the decomposition front velocity calculated with the input parameter $i$ equal to the mean plus $\tau$ standard deviations with all other input parameters evaluated at the mean.

By leveraging the 25 "level 1" response surface samples with an additional 25 functional evaluations (for a total of 51 evaluations), a "level 2" simple quadratic (with no cross terms) response surface can be formed to approximate the multivariate decomposition front velocity as follows:

$V_{Q \cup A D}=b_{o}+\sum_{i=1}^{25} b_{i}\left(\zeta_{i}-\bar{m}_{i}\right)+\sum_{i=1}^{25} c_{i}\left(\zeta_{i}-\bar{m}_{i}\right)^{2}$

VQUAD is the simple quadratic approximation of the decomposition front velocity fit to multivariate results from the 1-D finite element model. The $b$ 's and $c$ 's can be calculated as follows:

$b_{0}=V_{0}$

$b_{i}=0.5\left(V_{i}^{+\tau \sigma}-V_{i}^{-\tau \sigma}\right) /\left(\tau \sigma_{i}\right)$

$c_{i}=0.5\left(V_{i}^{+\tau \sigma}+V_{i}^{-\tau \sigma}-2 V_{o}\right) /\left(\tau \sigma_{i}\right)$

$V_{i}^{-\tau \sigma}$ is the decomposition front velocity calculated with the 1-D finite element model with the input parameter $i$ equal to the mean minus $\tau$ standard deviations with all other input parameters evaluated at the mean. More complex response surface expressions for $V$ can also be determined, however, the linear ap- 
proximation given in Eq. (7) and the simple quadratic approximation given in Eq. (11) were sufficient for this study.

With the simple analytical expression for the decomposition front velocity, a Monte Carlo evaluation can be performed without the expense of running the CPU-intensive model for each functional evaluation. Thus, the term "surrogate" means that the full finite element model is not used in the Monte-Carlo simulation; instead, an approximate response surface model is used for the evaluations of decomposition front velocity. Rather than using simple random sampling as in traditional Monte Carlo techniques, a more efficient, constrained sampling technique was used in the present work: Latin Hypercube Sampling (LHS). The LHS technique developed by McKay et al. ${ }^{9}$ selects $n$ different values for each of the 25 variables, $\zeta_{i}$. In this report, the number of samples, $n$, was selected to be 300 , which was determined to be sufficient, since a sample size of $300 ; 3,000 ; 30,000$; and 300,000 gave essentially the same results. The range of each input parameter is divided into $n$ non-overlapping intervals based on equal probability. One random value from each interval is selected according to the probability density function in the interval. The $n$ values thus obtained for $\zeta_{1}$ are paired in a random manner with the $n$ values obtained for $\zeta_{2}$. These $n$ pairs are then combined in a random manner with the $n$ values of $\zeta_{3}$ to form $n$ triplets, and so on, until $n$ sets of the 25 input variables are formed. The decomposition front velocity is then calculated $n$ times with the $n$ different sets of input parameters. The mean and standard deviation of decomposition front velocity are then calculated from the $n$ set of responses. More information on the LHS technique can be found in Ref. [10].

In this paper, the acronym LIN will represent the LHS analysis using the linear approximation of the decomposition front velocity, and QUAD will represent the LHS analysis using the quadratic approximation for the decomposition front velocity. To compare the LIN and QUAD results to the MV results discussed in the previous section, a direct LHS (DLHS) analysis was also performed. The DLHS analysis was performed by using the same Latin Hypercube sample used in the LIN and QUAD analysis; however, a full finite element analysis was used for each velocity evaluation rather than using the approximate equations to determine the decomposition front velocity. The DLHS technique should give the best approximation of the mean and standard deviation of the decomposition front velocity since no approximations are made regarding the linearity of the response surface.

Table 3 gives the mean and standard deviation of the decomposition front velocity calculated using the 51-run mean value (MV) technique, the 26-run Level 1 response surface technique (LIN), the 51-run Level 2 quadratic response surface technique (QUAD), and the direct LHS technique (DLHS) using 25, 50, and 300 samples. The mean and standard deviation in Table 3 were determined using either large or small time steps given previously in Table 2. To evaluate each of the different sensitivity/uncertainty models, the bias corrected mean, $\mu_{v}$, in Table 3 should be compared with the mean velocity, $\mu_{v}$, calculated with the DLHS method using 300 samples, which is boxed using double lines. Table 3 shows that all of the techniques give good estimates of the mean even with the larger unstable time steps. The MV technique gives a good estimate of the standard deviation when small time steps are used; however, the MV estimates of the standard deviation are not good when larger time steps are used. The problem with the MV method using larger time steps is related to the noisy derivative calculation discussed in the Numerical Issues section. The LIN and QUAD methods give good estimates even when the time step is large. These results were expected since the LIN and QUAD methods are not as sensitive to numerical noise as the MV method.

TABLE 3. MOMENTS OF DECOMPOSITION FRONT VELOCITY For $1,000^{\circ} \mathrm{C}$ RADIATION BOUNDARY CONDITION

\begin{tabular}{|c|c|c|c|c|c|c|c|}
\hline \multirow{2}{*}{$\begin{array}{l}\text { Element } \\
\text { size and } \\
\text { time step }\end{array}$} & \multirow[b]{2}{*}{$\begin{array}{c}\text { Moment, } \\
\mathrm{cm} / \mathrm{min}\end{array}$} & \multicolumn{6}{|c|}{ Method and number of functional evaluations } \\
\hline & & $\begin{array}{c}\mathrm{MV} \\
51\end{array}$ & $\begin{array}{l}\text { LIN } \\
26\end{array}$ & $\begin{array}{c}\text { QUAD } \\
51\end{array}$ & $\begin{array}{c}\text { DLHS } \\
25\end{array}$ & $\begin{array}{c}\text { DLHS } \\
50\end{array}$ & $\begin{array}{c}\text { DLHS } \\
300\end{array}$ \\
\hline \multirow{3}{*}{$\begin{array}{c}1-\mathrm{mm}, \\
\text { large time step }\end{array}$} & $\mu_{v}$ & 0.772 & 0.772 & 0.804 & 0.794 & 0.773 & 0.781 \\
\hline & bias corrected, $\mu_{v}$ & 1.093 & 1.093 & 1.125 & 1.115 & 1.094 & 1.102 \\
\hline & $\sigma_{v}$ & 0.550 & 0.091 & 0.107 & 0.115 & 0.103 & 0.106 \\
\hline \multirow{3}{*}{$\begin{array}{l}500-\mu \mathrm{m}, \\
\text { large time step }\end{array}$} & $\mu_{v}$ & 0.939 & 0.939 & 0.907 & 0.977 & 0.960 & 0.945 \\
\hline & bias corrected, $\mu_{v}$ & 1.093 & 1.093 & 1.061 & 1.131 & 1.114 & 1.099 \\
\hline & $\sigma_{V}$ & 0.488 & 0.115 & 0.121 & 0.134 & 0.130 & 0.126 \\
\hline \multirow{3}{*}{$\begin{array}{l}500-\mu \mathrm{m}, \\
\text { small time step }\end{array}$} & $\mu_{v}$ & 0.911 & 0.911 & 0.918 & 0.950 & 0.934 & 0.919 \\
\hline & bias corrected, $\mu_{v}$ & 1.093 & 1.093 & 1.100 & 1.132 & 1.116 & 1.101 \\
\hline & $\sigma_{v}$ & 0.116 & 0.110 & 0.117 & 0.129 & 0.125 & 0.120 \\
\hline \multirow{2}{*}{$\begin{array}{l}50-\mu \mathrm{m}, \\
\text { small time step }\end{array}$} & $\mu_{v}$ & 1.093 & 1.093 & 1.104 & 1.124 & 1.095 & 1.101 \\
\hline & $\sigma_{v}$ & 0.141 & 0.134 & 0.142 & 0.157 & 0.140 & 0.148 \\
\hline
\end{tabular}


The mean decomposition front velocities in Table 3 were corrected for discretization errors by using the grid and time-step independent MV velocity, $1.093-\mathrm{cm} / \mathrm{min}$. The discretization bias corrected velocities are also given in Table 3. A similar discretization bias correction was not necessary for the standard deviations of the decomposition front velocities indicating that the shape of the velocity distribution is independent of discretization errors. However, predictions of the standard deviation using the MV technique are sensitive to numerical noise.

The sensitivity of the standard deviation to numerical noise can be seen more readily in Fig. 5 where the mean and standard deviation of the decomposition front velocity is plotted as a function of the radiation boundary temperature. In Fig. 5 , results are from the MV, LIN, QUAD, and DLHS analysis obtained using $500-\mu \mathrm{m}$ elements and large time steps are plotted. All techniques give adequate estimates of the mean decomposition front velocity, although the QUAD velocity oscillates around the DLHS velocity. The oscillating velocity may be a result of neglecting cross terms in the surrogate surface approximation. The standard deviations calculated with the various methods are shown in Figs. 5.B and 5.C. In Fig. 5.B, the MV prediction of the standard deviation oscillates wildly due to the noisy derivative problem discussed previously. As shown in more detail in Fig. 5.C, the LIN and QUAD methods give good approximations to the DLHS method with the QUAD method being almost exact.

The MV method should not be used for uncertainty analysis unless the response surface is nearly linear, the solutions are extremely stable, and the functional evaluations are inexpensive. The least expensive uncertainty analysis in terms of CPU requirements is the LIN method. This method gives acceptable means and standard deviations. For more than about 25 uncertain variables, DLHS becomes competitive in terms of sampling efficiency. For example, both the 50 run and 25 run DLHS analysis shown in Table 3 gave means and standard deviations within a few percent of the converged 300-run results, which is competitive with the 51-run QUAD results.
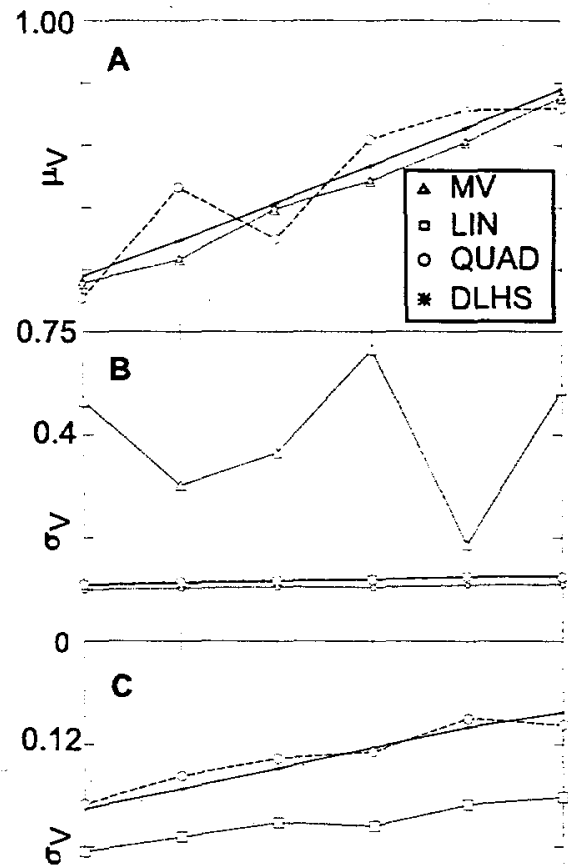

0.80 950 Radiation Temperature, ${ }^{\circ} \mathrm{C} \quad 1000$ Fig. 5. $\mu_{V}$ and $\sigma_{V}$ in $\mathrm{cm} / \mathrm{min}$

In LHS uncertainty/sensitivity analysis techniques, the most important factors are the parameters that cause the greatest deviations from the MV response variable. The importance of each factor, $\gamma_{i}^{2}$, can be obtained by:

$\gamma_{i}^{2}=\left(V_{0}-V_{i}^{ \pm \tau \sigma}\right) / V_{0}$

where $V_{0}$ is the decomposition front velocity calculated with the mean value of each input parameter and $V_{i}^{ \pm \tau \sigma}$ is the decomposition front velocity calculated with the input parameter $i$ equal to the mean $\pm \tau$ standard deviations with all other input parameters evaluated at the mean.

Table 4 shows the top three important factors as determined using the MV method, LIN method, and the QUAD

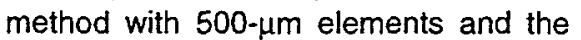
auto time step option. All of the analyses in Table 4 were performed using the input parameter mean and standard deviations given in Table 2 . For comparison, the top three important factors using the MV method with $50-\mu \mathrm{m}$ ele-

TABLE 4. IMPORTANCE FACTORS FOR $1,000^{\circ} \mathrm{C}$ BOUNDARY CONDITION

\begin{tabular}{|l|c|c|c|}
\hline Method, Element & \multicolumn{3}{|c|}{ Importance Ranking } \\
\cline { 2 - 4 } size, and time step & 1 & 2 & 3 \\
\hline MV, 500- $\mu \mathrm{m}$, large & $h_{r}$ & $\sigma+1$ & $\varepsilon$ \\
\hline LIN, 500- $\mu \mathrm{m}$, large & $C_{p}$ & $\rho_{0}$ & $\varepsilon$ \\
\hline QUAD, 500- $\mu \mathrm{m}$, large & $C_{p}$ & $\rho_{0}$ & $\varepsilon$ \\
\hline DLHS, 50- $\mu \mathrm{m}$, small & $C_{p}$ & $\rho_{0}$ & $\varepsilon$ \\
\hline
\end{tabular}

ments and the constrained time step option is also given. The LIN method - requiring 26 functional evaluations - gives the same results as the more expensive QUAD method that required 51 functional evaluations. The MV method using the large 
auto time step option - requiring 51 functional evaluations to determine the derivative of the response function - did not give the correct importance factors.

\section{SuMmaRY AND CONCLUSIONS}

Aristotle ${ }^{11}$ noted that "... it is the mark of an educated mind to rest satisfied with the degree of precision which the nature of the subject admits and not to seek exactness where only an approximation is possible." Precise, accurate input parameters will not make a model match experimental data when the model lacks important physical phenomena. For example, the foam decomposition model addressed in this paper does not consider mass transport limitations, species diffusion, bubble mechanics, fluid flow, gravitational effects, or mechanical response such as thermal expansion. Not including these physical phenomena may be acceptable for certain experimental conditions, such as when samples are small or when the heating rates are high. Under these conditions, diffusion lengths are small and mass transport limitations are negligible. For conditions that favor thicker decomposition fronts, such as confinement and/or low heating rates, the foam decomposition model should be improved with additional physics. At the same time, however, the importance of the driving contributions to uncertainty in any endeavor should be ascertained. For the base model analyzed here, the standard deviation of the response due to uncertainties in the 25 input parameters is on the order of $10 \%$ of the nominal computed results. Since nominal error in the model due to using $500-\mu \mathrm{m}$ vs. $50-\mu \mathrm{m}$ elements is also on the order of $10 \%(2-16 \%)$, the error from using $500-\mu \mathrm{m}$ elements is significant and some form of discretization bias correction should be used.

Sensitivity/uncertainty analyses on complex engineering models can be frustrating and time consuming. For example, the MV sensitivity/uncertainty analysis was repeated numerous times when the elements and/or time steps were found to be too large to compute accurate finite difference derivatives, resulting in thousands of additional functional evaluations to establish converged results. A lot of frustration could have been avoided if a thorough study of the problem numerics were initiated before beginning the sensitivity/uncertainty analysis. Another source of frustration is lack of information regarding the uncertainty associated with the various input parameters. Variability in input parameters should be obtained and verified experimentally.

Three sensitivity analyses (MV, LIN, and QUAD) were compared to a direct LHS (DLHS) simulation. The analytical MV technique required the derivative of the decomposition front velocity for each input parameter resulting in 51 functional evaluations. The MV uncertainty results were also shown to be extremely sensitive to numerical noise. The LIN method required only 26 functional evaluations, was found to be relatively insensitive to numerical noise, and gave acceptable predictions of the mean and standard deviation. The QUAD method required 51 functional evaluations, was found to be nearly insensitive to numerical noise, and overall gave the closest values of mean and standard deviation to the converged DLHS results. However, the mean velocities predicted with the QUAD method were subject to oscillation, which may have been a result of neglecting cross-terms in the surrogate surface approximation. Various sample sizes were used for the DLHS. If the number of random variables is substantially more than 25 variables, a DLHS analysis is highly recommended.

\section{ACKNOWLEDGEMENTS}

Discussions regarding sensitivity analysis with D. G. Robinson, and DAKOTA support from M.S. Eldred are gratefully acknowledged. Internal reviewers at Sandia National Laboratories, B.F. Blackwell and B.L. Oberkampf provided insightful suggestions for improvement of this paper.

\section{References}

1. Gartling, D.K., R.E. Hogan, M.W. Glass (1998), "COYOTE - A Finite Element Computer Program for Nonlinear heat Conduction Problems, " Version 3.0, Part 1 - Theoretical Background: SAND94-1173, Part2 - User's Manual: SAND94-1179, Sandia National Laboratories, Albuquerque NM.

2. Chu, T.Y., M.L. Hobbs, K.L. Erickson, T.A. Ulibarri, A.M. Renlund, W. Gill, L.L. Humphries, T.T. Borek (1999), "Fire Induced Response in Foam Encapsulants," 44 $4^{\text {th }}$ intemational SAMPE Symposium \& Exhibition, Long Beach, CA. Proceedings.

3. Hobbs, M.L. K.L. Erickson, and T.Y. Chu (2000), "Modeling Decomposition of Unconfined Rigid Polyurethane Foam, Polymer Degradation and Stability, 69, 47 (2000).

4. Robinson, D. G., "A Survey of Probabilistic Methods Used in Reliability, Risk and Uncertainty Analysis: Analytical Techniques," Sandia National Laboratories Report SAND98-1189, Albuquerque, NM (1998). 
5. Romero, B. J. and Bankston, S. D., "Efficient Monte Carlo Probability Estimation with Finite Element Response Surfaces built from Progressive Lattice Sampling," $39^{\text {th }}$ SDM (Structures, Structural Dynamics and Materials) Conference, Long Beach, CA, AlAA98-1826 (1998).

6. Romero, V. J. and Bankston, S. D., "Finite-Element/Progressive-Lattice-Sampling Response Surface Methodology and Application to Benchmark Probability Quantification Problems," Sandia National Laboratories Report SAND98-0567, Albuquerque, NM (1998).

7. Romero, V. J., "A Systematic Approach to Non-Deterministic Analysis with Large Computer Models," Sandia National Laboratories Report, Albuquerque, NM (in review).

8. Guan, X. L. and Melcher, M., "A Parametric Study on the Response Surface Method," Proceedings of the $8^{\text {th }}$ ASCE Joint Specialty Conference on Probabilistic Mechanics and Structural Reliability, PMC2000-023, Notre Dame, IN (2000).

9. McKay, M. D., Conover, W. J., and Beckman, R. J., "A Comparison of Three Methods for Selecting Values of Input Variables in the Analysis of Output from a Computer Code," Technometrics, 221, 239 (1979).

10. Wyss, G. D., Jorgensen, K. H., "A User's guide to LHS: Sandia's Latin Hypercube Sampling Software," Sandia National Laboratories Report SAND98-0210, Albuquerque, NM (1998).

11. Aristotle, quoted in The Daily News, Sandia National Laboratories (January 7,1999 ). 